\title{
Non destructive testing of works of art by stimulated infrared thermography
}

\author{
By Jean Charles Candoré ${ }^{*}$ J.L Bodnar*, Vincent Detalle**, Philippe Grossel*
}

*Laboratoire d'Energétique et d'Optique, UFR Sciences Exactes et Naturelles, BP 1039, 51687 Reims cedex 02

** Laboratoire de recherche des monuments historiques, 29 avenue du Paris, 77420 Champs sur Marne

\section{Abstract}

As part of the assistance to the restoration of the works of art, the Resarch Laboratory of Historical Monuments (LRMH), the Research Restoration Center of the Museums of France (C2RMF) and the restorers use many methods, physical and chemical instruments. As example, we can quote visible photography, photography ultra-violet, infra-red photography (implementated in natural light, polarized light or in low-angled light), radiography $X$, electronic scan microscopy, the spectroscopy, chromatography in liquid phase or vapor, the particle accelerator AGLAE, the acoustic sounding,... These various methods are very powerful in their scopes of application but are not universal. In addition, certain methods can be difficult to implement. For example, the detection of defects in mural paintings (being able to extend in the churches on a very important surface) by acoustic analysis (by knoking the surface with finger).

New control methods, destructive or not can thus still find their place in the field of the assistance to the restoration of works of art.

The Laboratory of Energetics and Optics of the University of Reims Champagne Ardennes has worked for approximately 20 years, with the development of a particular method of non destructive testing : photothermal radiometry. The principle of this method of analysis is relatively simple : It consists in subjecting the sample to be analyzed with a luminous flow whose absorption produces a local rise in the temperature close to the point of luminous impact, then to observe the variations of flux densities of material using a chain of infra-red optical detection. The photothermic signal thus obtained depends on the optical and thermophysical properties of analyzed material, it is possible then to characterize it. This is a nondestructive test, without contact, used for the thermophysical analysis of thin material. It was already implemented to detect and characterize various types of localised or extended defects (delaminations, cracks, inclusions,...), in various types of materials. It seems to be able to be used in the field of the restoration of works of art, for example helping with the restoration of mural paintings, in addition to acoustic survey. That explains why for now several years, we used it in this way. In this work, various examples of assistance to the restoration of works of art by stimulated infra-red thermography are presented.

We show initially the possibility to detect by this technique, separations or delaminations in frescoes, such as for example in the Saint Christophe of the Campana collection of Louvres or on the mural paintings of the church Saint Florentin de Bonnet.

We show then the possibility to detect by this technique, separations, delaminations or galleries of worms in marquetries.

We still show the possibilities to detect, by this technique, the boilings or separations of greyness in stained glasses, such as for example those of the Cathedral of Chartres.

We show in a fourth stage, the possibility to obtain, thanks to this technique, an imagery of the internal structure of the ceilings of the castle of Champs-sur-Marne.

We show finally the possibility to detect, thanks to this technique, the cracks in ceramics.

9th International Conference on Quantitative InfraRed Thermography

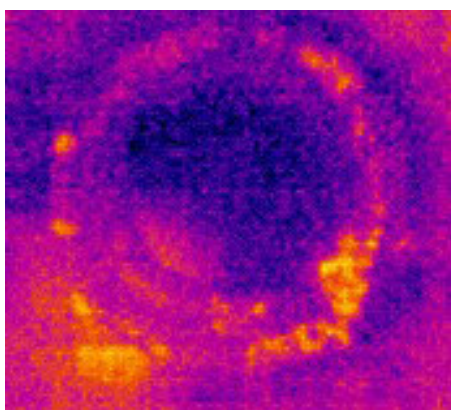

Fig.1. Detection of boiling and detachment of greyness in a stained glass 


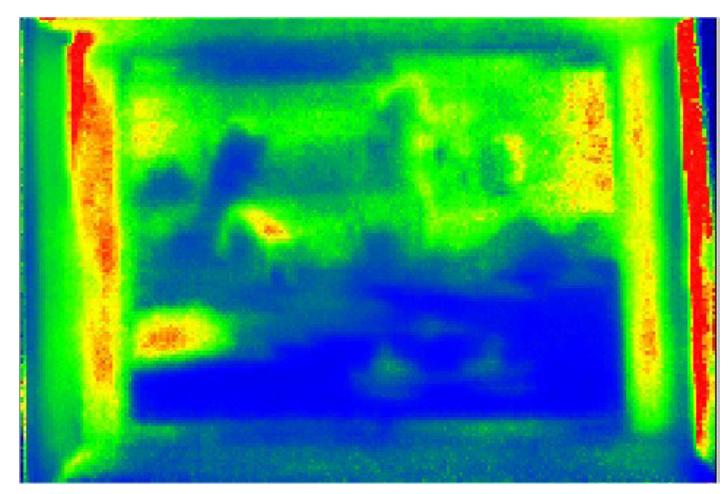

Fig.2. Detection of delaminations in a marquetry

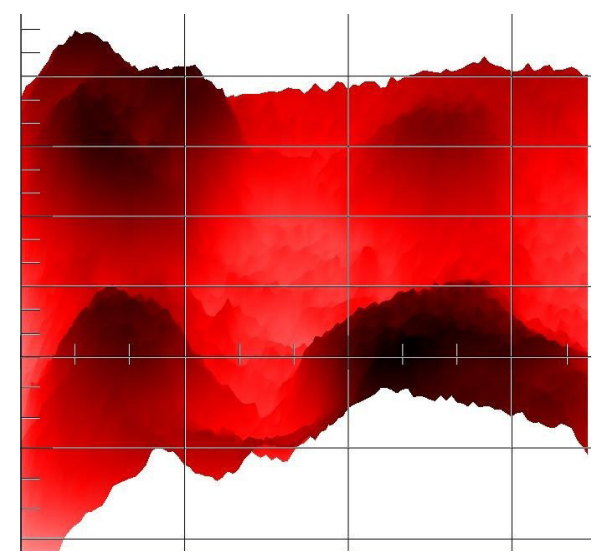

Fig.3. Detection of four delaminations in a fresco

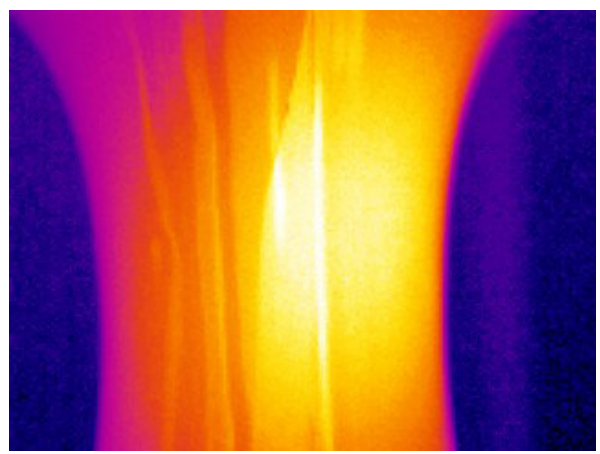

Fig.4. Detection of a crack in an ovoid ceramics vase 